\title{
基于深度强化学习的舰载机动态避障方法
}

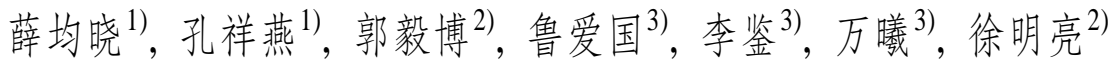 \\ 1) (郑州大学软件学院 郑州 450002) \\ 2) (郑州大学信息工程学院 郑州 450001) \\ 3) (中国船舶重工集团第 709 研究所 武汉 430070) \\ (iexumingliang@zzu.edu.cn )
}

\begin{abstract}
摘 要: 针对高度异构、动态的航母甲板作业场景中的舰载机避障问题, 提出一种结合预测算法和深度强化学习的 避障方法. 该方法包含场景建模、奖励模型和轨迹预测模型等模块. 首先基于智能体状态和动作空间对航母板场景 进行建模; 然后利用最小二乘法对场景中动态障碍物的位置进行实时轨迹预测, 并构造了包含路径预测模块的深度 强化学习方法——环境预测深度 Q 网络(PDQN); 最后利用该方法实现航母甲板作业场景中的舰载机动态避障. 利用 Python 绘图集 Matplotlib 进行仿真实验, 实验数据结果表明, 相比于 Q-learning, SARSA 等方法, 所提方法的准确率 提升了 15\% 25\%, 路径长度短 9\% 39\%, 平均奖励值高 30\% 100\%, 收玫速度快 1 2 倍且训练平稳后准确率的标准 差小 $2 \% \sim 50 \%$.
\end{abstract}

关键词：航空母舰; 强化学习; 轨迹预测; 动态避障

中图法分类号: TP391.41

DOI: $10.3724 /$ SP.J.1089.2021.18637

\section{Dynamic Obstacle Avoidance Method for Carrier Aircraft Based on Deep Reinforcement Learning}

\author{
Xue Junxiao ${ }^{1)}$, Kong Xiangyan ${ }^{1)}$, Guo Yibo ${ }^{2)}$, Lu Aiguo ${ }^{3)}$, Li $\operatorname{Jian}^{3)}$, Wan $\mathrm{Xi}^{3)}$, and Xu Mingliang ${ }^{2)}$ \\ 1) (School of Software Engineering, Zhengzhou University, Zhengzhou 450002) \\ 2) (School of Information Engineering, Zhengzhou University, Zhengzhou 450001) \\ 3) (No.709 Research Institute of China Shipbuilding Industry Corporation, Wuhan 430070)
}

\begin{abstract}
Aiming at the obstacle avoidance problem of carrier aircraft in the highly heterogeneous and dynamic aircraft carrier deck operation scene, a deep reinforcement learning obstacle avoidance method combined with a prediction algorithm is proposed. The method includes scene modeling, reward model and trajectory prediction model. First, the aircraft carrier deck scene is modeled based on the agent state and action space. Then the least square method is used to predict the position of dynamic obstacles in the scene in real-time and a deep reinforcement learning algorithm — environmental prediction deep Q network (PDQN) is constructed which includes a path prediction module. Finally, the algorithm is used to achieve dynamic obstacle avoidance in the aircraft carrier deck operation scene. The Python drawing set Matplotlib is used for simulation experiments. The experimental results show that, compared with Q-learning, SARSA, the accuracy of the proposed method is improved by $15 \%-25 \%$, the path length is shorter by $9 \%-39 \%$, the average reward
\end{abstract}

收稿日期: 2020-09-04; 修回日期：2021-02-10. 基金项目：国家自然科学基金(62036010，61822701); 河南省高校科技创新人才 支持计划(18HASTIT020). 薛均晓(1982一), 男, 博士, 副教授, 硕士生导师, CCF 会员, 主要研究方向为人工智能、虚拟现实; 孔祥燕 (1996-), 女, 硕士研究生, 主要研究方向为强化学习、路径规划; 郭毅博(1987一), 男, 博士, 讲师, 硕士生导师, 主要研究方向为机 器学习; 鲁爱国(1979一), 男, 学士, 研究员, 主要研究方向为人工智能; 李鉴(1969一), 男, 学士, 研究员, 主要研究方向为机器视 觉; 万䂀(1981一), 女, 学士, 高级工程师, 主要研究方向为机器学习; 徐明亮(1981一), 男, 博士, 教授, 博士生导师, CCF 会员, 主 要研究方向为计算机图形学、人工智能. 
value is higher by $30 \%-100 \%$, the convergence speed is $1-2$ times faster, and the standard deviation of the accuracy after training is small by $2 \%-50 \%$.

Key words: aircraft carrier; reinforcement learning; trajectory prediction; dynamic obstacle avoidance

航母甲板空间狭窄，作业任务多，是一个高度 异构、动态的危险作业场景. 要在高度受限的空间 内, “人-机-物”混杂分布的环境下, 确保甲板作业 的高效和安全开展, 需要解决航母甲板上作业要 素之间的路径规避问题.

在航母甲板上，作业区域存在部分重合的情 况, 舰载机在当前时刻进行 $A$ 作业, 在另一时刻进 行 $B$ 作业. 且航母甲板上没有固定的作业通道, 因 此增加了甲板上路径规划的难度.甲板路径规划 和一般的路径规划问题不同，后者只强调规划时 间和效率，不过多考虑高密度的情况，而前者须将 两者都重视起来. 若舰载机路径规划时间短效率 高, 舰载机出动能力得到提升, 航母作战能力得到 增强; 若舰载机无法在高密度高动态情况下进行 路径规划, 找不到合理的调运路径, 将影响航母的 作战能力. 由于舰载机的调运是顺序排列的, 所以 在有限的空间内, 需要规划出合理的距离才不会 导致其他甲板作业延误. 由于舰面同时存在多个 机务保障点, 会出现多架舰载机同时出现调运的 情况, 即多车多机同时作业的动态场景. 因此, 不 仅要考虑舰载机与船体的碰撞, 还要考虑动态舰 载机之间的碰撞.

强化学习可以用来解决类似的决策问题, 且 相关研究有着悠久的发展史 ${ }^{[1-10]}$, 从 Bellman ${ }^{[1]}$ 提出 的动态规划到 $\mathrm{Q}$-learning ${ }^{[4]}$, 状态-动作-奖励-状态动 作 (state-action-reward-state-action, SARSA) ${ }^{[5]}$, 强化 学习逐渐发展成熟. 此后监督学习的出现, 使强化 学习的发展变得十分缓慢. 2015年, DeepMind 团队 提出了深度强化学习 ${ }^{[11]}$ (deep Q-learning, DQN)算 法, 标志着深度强化学习的出现, 至此强化学习开 始了新的篇章. 随后该团队又提出计算机围棋初 栾号 ${ }^{[12]}$ 和 $\mathrm{A} \mathrm{C}^{[13]}$. 深度强化学习取得了巨大的进 步, 成为解决高动态场景下实时避障问题的一种 可能方案.

\section{1 相关工作}

路径规划的目的是在有障碍的环境中找到一 条最优或次优的无碰撞路径. 早期路径规避大多是
基于图的路径规划方法 ${ }^{[14-17]}$, 如可视图、C 空间 ${ }^{[17]}$ 等, 但这些方法多用于静态环境, 无法解决动态障 碍物的问题. 相比之下, 人工势场 ${ }^{[18]}$ 、扩展快速随 机搜索树 ${ }^{[19]}$ 、动态 $A^{*}{ }^{[20]}$ (dynamic $A^{*}, D^{*}$ ) 是典型的 用于动态环境下的路径规划方法. 人工势场结构 简单且有良好的实时性, 但该方法容易陷人局部 最小值而无法到达目标点. 快速随机搜索树在连 续高动态的环境中效果不佳. $\mathrm{D} *$ 无法应对最短路 径上距离智能体较远节点上发生的变化.

强化学习作为一个新技术的出现, 赋予智能体 充足的智能去做决策和完成任务. 2011 年, Jaradat 等 ${ }^{[21]}$ 利用 Q-learning 对于动态环境中机器人的路径 规划进行研究, 之后 Yan 等 ${ }^{[22]}$ 和 Bouhamed 等 ${ }^{[23]}$ 将 Q-learning 应用到无人机的路径规划中. Q-learning 在此类静态或低动态场景下有着很好的表现, 然 而在高动态、高密度且复杂的场景中, 由于维度爆 炸, Q-learning 很难收玫.

近些年, 人工智能技术正在快速发展和应用. 结合深度学习和强化学习的深度强化学习为复杂 环境下的感知决策问题提供了解决方案, 利用神 经网络代替 $\mathrm{Q}$ 表格, 解决了 Q-learning 的维度爆炸 的问题. 2017 年, Chen 等 ${ }^{[24]}$ 结合强化学习和社会意 识实现了智能体遵循某种特定的行为规则制定路 径规划的目标. 2018 年, Everett 等 ${ }^{[25]}$ 提出了一种结 合深度强化学习和长短期记忆网络的路径规划方 法, 其需要进行大量训练. 2019 年, Tang 等 ${ }^{[26]}$ 提出 了一种基于浅试规则的深度强化学习的方法, 其 对于静态、低动态环境效果比较好, 但在高动态环 境下难以收玫.

由于航母甲板作业场景的特殊性，没有可获 得的预期信息和数据. 因此, 为了解决无数据训 练、传统方法泛化能力差、收玫速度慢以及大量动 态障碍物造成的环境复杂等问题, 针对航母甲板 上动态避障, 本文提出了一种基于深度强化学习 的舰载机动态避障方法. 该方法结合深度强化学 习算法和轨迹预测算法, 同时在深度强化学习中 融人动态奖励模块.

本文的主要工作和创新性如下.

（1）提出了一种异构、动态场景的避障方法— 
环境预测深度 Q 网络 (environmental prediction deep Q network, PDQN), 该方法在深度强化学习 方法中加人预测模型, 用于动态障碍物的轨迹预 测, 并通过有效的奖励机制实现了航母甲板作业 场景中多要素之间的路径规避.

（2）基于智能体状态和动作空间对航母甲板 场景进行了仿真建模, 并利用该仿真模型验证了 文中提出的避障方法. 实验结果显示, 该方法在准 确率、路径长度、收玫速度、平均奖励值和稳定性 上都有很好的表现.

\section{2 本文方法}

\section{1 场景建模}

在航母甲板建模中，将障碍物分为动态障碍 物和静态障碍物, 将停靠位置、起飞位置等设置为 作业点, 作业时间随机生成. 舰载机的任务是利用 PDQN 方法找到一条从起点到目的地尽可能短的 无碰撞路径.

\subsection{1 障碍物设置}

障碍物分为动态障碍物和静态障碍物. 静态 障碍物指舰载机在寻径的过程中, 一直静止在初 始位置上的障碍物. 动态障碍物指在舰载机寻径 的过程中会发生运动的障碍物 (正在或即将做任务 的舰载机), 其状态在临时静态和临时动态间转换. 当障碍物从甲板上一个作业点向另一个作业点运动 时其状态为临时动态, 障碍物进行作业时其状态为 临时静态. 开始时, 所有动态障碍物的状态默认为 临时动态, 从预先设定好的初始位置 $\left(p_{x}^{0}, p_{y}^{0}\right)$, 以 初始速度 $\left(v_{x}^{0}, v_{y}^{0}\right)$ 开始运动, 当障碍物运动到作业 位置 $\left(p_{x}^{t}, p_{y}^{t}\right)$ 时, 其状态更改为临时静止, 在此位 置上进行随机短时间作业, 作业完成后, 其状态变 为临时动态且随机生成新的速度 $\left(v_{x}, v_{y}\right)$.

\subsection{2 智能体状态和动作空间}

智能体的状态空间表示智能体在决策之前从 环境中获得的信息, 可以帮助智能体更好地了解 当前环境状态. 而智能体的动作空间则表示智能 体根据状态空间所决定要执行的动作集合.

本文中智能体是全局可观测，即可观测到整 个环境的信息. 根据障碍物的历史轨迹, 通过预测 模块预测障碍物下一步的位置, 利用障碍物的预 测位置, 当前智能体舰载机的位置和目标点的位 置设计状态 $s$; 然后将状态值输人 PDQN, 计算智 能体的动作.
定义环境的状态为

$s=\left(\{x, y\},\left\{x^{1}, y^{1}\right\}, \cdots,\left\{x^{n}, y^{n}\right\},\left\{x^{1}, y^{1}\right\}, \cdots,\left\{x^{m}, y^{m}\right\}\right)$. 其中, $x$ 和 $y$ 分别表示舰载机和目标点在横纵坐 标上的距离差在环境中的占比; $x^{i}$ 和 $y^{i}$ 分别表示 静态障碍物和舰载机在横纵坐标上的距离差在环 境中的占比; $x^{\prime j}$ 和 $y^{\prime j}$ 分别表示动态障碍物和舰 载机在横纵坐标上的距离差在环境中的占比; 它 们计算公式分别为

$$
\begin{aligned}
& x=\frac{x_{p}-x_{o}}{W}, y=\frac{y_{p}-y_{o}}{H} ; \\
& x^{i}=\frac{x_{o}^{i}-x_{p}}{W}, y^{i}=\frac{y_{o}^{i}-y_{p}}{H}, \quad i \in(1, n) ; \\
& x^{\prime j}=\frac{x_{l}^{j}-x_{p}}{W}, y^{\prime j}=\frac{y_{l}^{j}-y_{p}}{H}, j \in(1, m) .
\end{aligned}
$$

其中, $\left(x_{p}, y_{p}\right)$ 表示舰载机中心点的坐标; $\left(x_{o}, y_{o}\right)$ 表示目标点的坐标; $\left(x_{o}^{i}, y_{o}^{i}\right)$ 表示静态障碍物 $i$ 的 中心点的坐标; $\left(x_{l}^{j}, y_{l}^{j}\right)$ 表示动态障碍物 $j$ 预测位 置中心点的坐标; $W$ 和 $H$ 分别表示环境的宽和高.

定义智能体的动作集为 $A=\left\{a_{u}, a_{d}, a_{l}, a_{r}\right\}$. 其 中, $a_{u}, a_{d}, a_{l}, a_{r}$ 分别表示舰载机向上、向下、向 左、向右移动的决策.

\subsection{PDQN 方法}

本文提出了一种基于深度强化学习的舰载机 动态避障方法, 其框架如图 1 所示. 在每个时间步 $t_{i}$, 环境将状态 $s$ (包括动态障碍物的预测位置、静 态障碍物的位置和目标舰载机的位置)输人 PDQN 的主干网络, 再根据网络输出并利用 $\varepsilon$-greedy 策略 选择动作 $a$ 作用于环境. 环境执行动作 $a$ 后, 其状 态变为 $s^{\prime}$, 且将 $\left(s, a, r, s^{\prime}\right)$ 存储在经验池中, 同时将 所有动态障碍物在 $t_{i}$ 时刻的动作作为各自历史轨迹 输给预测模块. 主干网络和目标网络从经验池中分 别获取一批数据用于计算损失函数, 根据误差函数 的梯度 $\Delta \theta$ 更新主干网络. 每隔 $N$ 个时间步, 将主 干网络的网络参数复制目标网络.

\subsection{1 轨迹预测模型}

PDQN 的预测模块流程如图 2 所示. 首先获取 环境信息, 判断舰载机周围的障碍物是否为动态 障碍物, 如果是, 利用最小二乘法预测其位置; 如 果不是, 则直接获取当前位置. 然后利用获取到的 位置信息生成状态值, 将其输人 DQN 中做出决策 并执行. 最后判断舰载机是否到达终点, 到达终点 则结束, 否则跳回第 1 步重新获取环境信息. 结合 航母场景的特殊性做以下假设. 


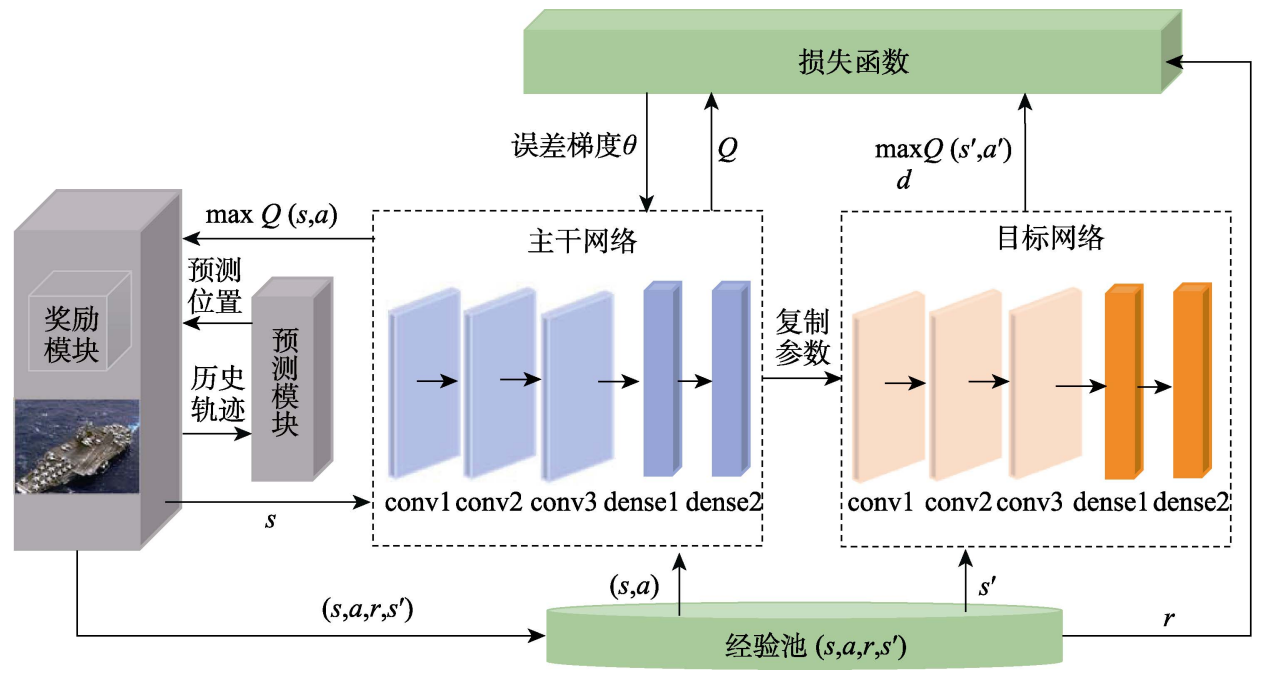

图 1 PDQN 方法框架

假设 1: 障碍物的运动有目的.

假设 2: 设置甲板上特殊位置(如加油站、指挥 室等)为作业区域.

假设 3: 给障碍物一个初始速度, 当障碍物运 动到特定位置(作业区域)时，在此位置上随机停留 几秒进行作业, 完成后随机生成障碍物速度 $\left(v_{x}, v_{y}\right)$, 并定义 $x$ 方向上的运动轨迹为

$$
f(x)=a_{0}+a_{1} x+a_{2} x^{2}+\cdots+a_{k} x^{k} .
$$

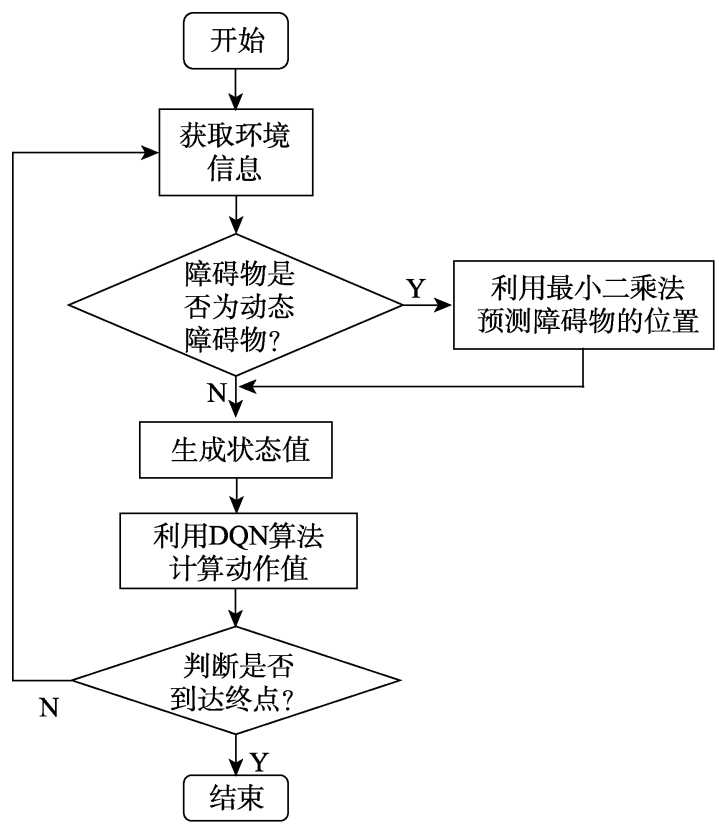

图 2 预测模块的流程

\subsection{2 基于 PDQN 的奖励模型}

PDQN 的奖励值 $r_{t}$ 是一个标量反馈信号，其衡 量智能体在时刻 $t$ 所执行动作 $a$ 的表现，智能体的 目标是最大化累计奖励值. 奖励设置的好坏决定
了智能体最终是否能学到期望的技能, 并直接影 响该方法的收玫速度和最终性能.

为了设计出动态奖励模型, 定义了奖励函数, 对不同的状态定义如下.

成功状态：舰载机处于目标位置.

失败状态：舰载机和障碍物发生碰撞。

普通状态：舰载机处于除目标位置和障碍物 位置之外的甲板上的所有位置.

危险状态：舰载机任意移动后和障碍物预测 轨迹发生碰撞的状态.

各个状态奖励值的设置如下所示.

成功状态: 当 $p_{f}=p_{t}$ 时, $r=r+100$. 其中, $p_{f}$ 表示舰载机当前位置, $p_{t}$ 表示目标位置.

失败状态：如图 3 所示，当 $D>d$ 时，如图 $3 a$ 所示为安全行驶状态; 当 $D<d$ 时, 如图 3b 所示 为碰撞状态. 当 $\exists D<d$ 时, $r=r-20$. 其中, $d$ 表 示包围盒的边长. $D=\left\{\left|p, p_{o j}^{\prime}\right| \mid j=1,2, \cdots, n\right\} ; \quad p$ 表 示舰载机当前的位置; $p_{o j}^{\prime}$ 表示障碍物中的任意障 碍物, $j \in(1, n)$.

普通状态: 当 $d_{s o}>d_{s^{\prime} o}$ 时, $r=r+1$; 当 $d_{s o}<d_{s^{\prime} o}$ 时, $r=r-1$. 其中, $d_{s^{\prime}}$ 表示当前舰载机离目标位 置的距离; $d_{s o}$ 表示上一时刻舰载机离目标位置的 距离.

危险状态: 当 $\exists D_{1}<d$ 时, $r=r-5$. 其中, $D_{1}=\left\{\left|p_{f i}, p_{o j}^{\prime}\right| \mid i=1,2,3,4 ; j=1,2, \cdots, n\right\} ; d$ 表示包 围盒的边长; $p_{f 1}, p_{f 2}, p_{f 3}, p_{f 4}$ 分别表示舰载机 执行向上、向下、向左、向右的位置; $p_{o j}^{\prime}$ 障碍物 中的任意障碍物, $j \in(1, n)$. 


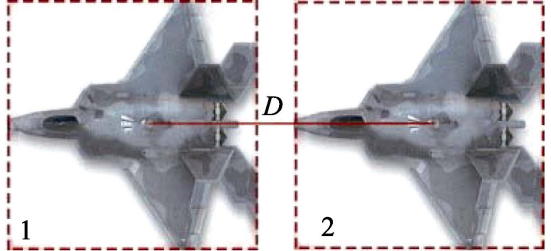

a. 安全行驶状态

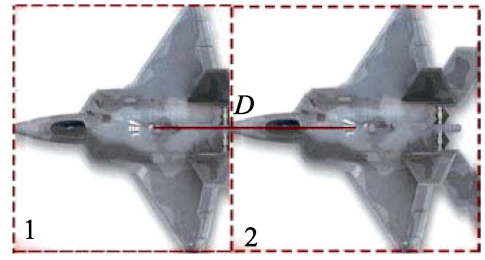

b. 碰撞状态

图 3 碰撞检测图

动态奖励模块流程如图 4 所示, 首先利用 DQN 决策舰载机的动作并执行; 然后判断舰载机 是否处于失败状态. 若是, 则 $r=r-20$, 结束; 否 则判断其是否更靠近终点, 如果更靠近, 则 $r=r+1$, 反之 $r=r-1$. 接着判断其是否处于危险 状态，如果满足，则 $r=r-5$, 最后判断是否满足 成功状态, 若满足 $r=r+100$, 则结束; 否则返回第 1 步重新选择动作.

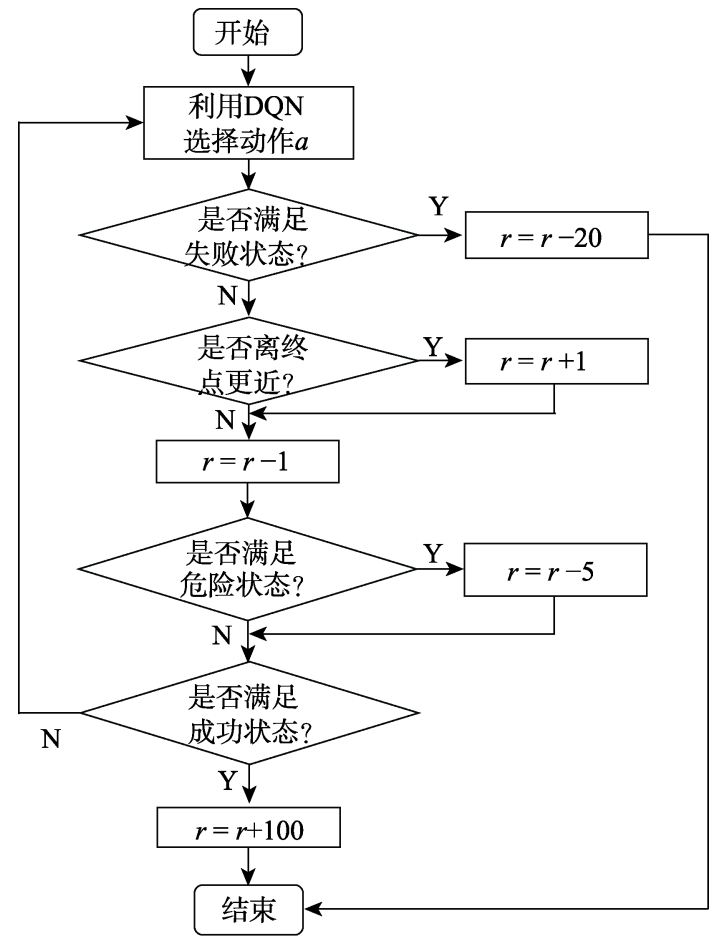

图 4 动态奖励模块流程

\section{3 仿真分析与验证}

利用 Python 的绘图集 Matplotlib 进行仿真, 它
可以与 NumPy 一起使用, 提供了一种有效的 Matlab 开源替代方案. 运行环境为 Intel i7 8700K 6 核 $3.7 \mathrm{GHz}$ 主频, GeForce RTX 2070 SUPER.

为了保证 PDQN 在大量动态障碍物中仍能保 持较高的准确性, 可以通过增加动态障碍物的数 量设置多个实验场景, 从准确率、路径长度、收玫 速度、平均奖励值和稳定性上, 用 PDQN 以及 Q-learning, SARSA 和 DQN 进行比较. 准确率指舰 载机从起点开始不碰到任何障碍物成功到达目的地 的概率, 准确率越高, 越有效, 其计算公式为 $T_{r}=\sum_{i=1}^{100} u / 100$. 当舰载机无碰撞到达终点时, $u=1$; 否则 $u=0$.

路径长度是直接反映路径规划方法质量的重 要评价指标, 为避免方法偶然性带来的误差, 随机 抽取 100 组数据计算其平均路径长度. 收玫速度是 指一个迭代序列向其局部最优值逼近的速度，反 映自学习能力. 奖励值 $r_{t}$ 是一个标量反馈信号, 其 衡量智能体在时刻 $t$ 所执行动作 $a$ 的表现. 每 100 步计算一次平均奖励值, 平均奖励值越高, 表示舰 载机选择的路线越短、越合理. 标准差指的是总体 各单位标准值与其平方的算术平均数的平方根, 它反映组内个体间的离散程度. 为衡量方法的稳 定性, 随机抽取训练稳定后的 80 组准确率的数据, 计算其标准差.

\section{1 参数设置}

参数的设置很大程度上会影响实验结果，为 公平起见, 4 种方法设置相同的参数, 具体的参数 设置如表 1 所示.

表 1 参数设置

\begin{tabular}{llr}
\hline \multicolumn{1}{c}{ 参数 } & \multicolumn{1}{c}{ 含义 } & 大小 \\
\hline$\gamma$ & 衰减因子 & 0.900 \\
learning-rate & 学习率 & 0.011 \\
num-action & 动作数量 & 4.000 \\
memory-size & 经验池大小 & 2000.000 \\
$\varepsilon$ _greedy & $\varepsilon$ _greedy 策略参数 & 0.900 \\
replay-target-iter & 目标网络更新步数 & 300.000 \\
batch_size & minibatch 大小 & 32.000 \\
num-train & 训练步数 & 25000.000 \\
\hline
\end{tabular}

\section{2 仿真结果}

3.2.1 场景描述和仿真结果

在仿真系统中设计了 3 个不同的网格场景, 且 为了安全起见, 使用包围盒理论实现碰撞检测. 如 
图 5 所示, 对障碍物和舰载机使用不同颜色的包围 盒, 起点和终点分别为黑色包围盒和绿色圆点所 在的位置. 静态障碍物、动态障碍物和目标舰载机 分别为蓝色、红色和黑色包围盒中的舰载机. 3 个 场景起点和终点相同, 障碍物数量和类型不同, 将 特定的地点为作业地点, 作业时间随机生成. 如图 $5 \mathrm{a}$ 所示, 场景 1 中, 设置 3 个静态障碍物和 2 个动 态障碍物, 障碍物在起点和终点周围以增加避障 的难度; 如图 5b 所示, 相比于场景 1 , 场景 2 中的 障碍物的位置不变, 部分静态障碍物变为动态障
碍物; 如图 5c 所示, 对于场景 3, 在场景 2 的基础 上, 增加 2 个静态障碍物. 图 6 所示为场景 3 中 PDQN 的仿真图. 如图 6a 所示, 舰载机 $b$ 比其他舰 载机晚一个时间步开始运动. 当 $t=2$ 时, 舰载机 $h$ 到达作业地点. 当 $t=3$ 时, 舰载机 $e$ 到达作业地 点. 当 $t=5$ 时, 舰载机 $b$ 到达作业地点. 舰载机 $g$ 一直在目标舰载机 $f$ 后面. 如图 6c 所示, 为躲避舰 载机 $e$, 目标舰载机 $f$ 在 $t=8$ 时, 才执行向上的动 作. 如图 6d 所示, $t=11$ 时, 舰载机 $f$ 成功地躲避所 有动静态障碍物并以最短路径到达目标点.

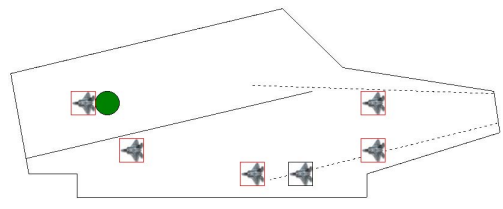

a. 场景 1

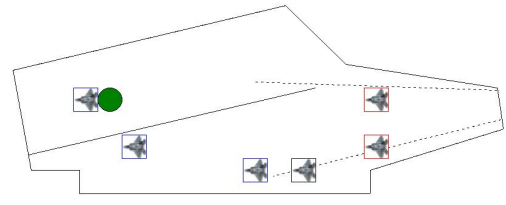

b. 场景 2

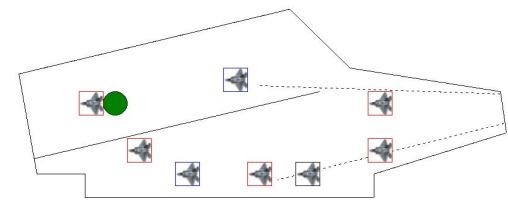

c. 场景 3

图 53 个场景初始状态

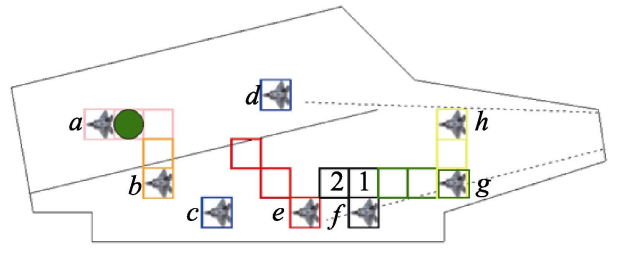

a. $t=2$

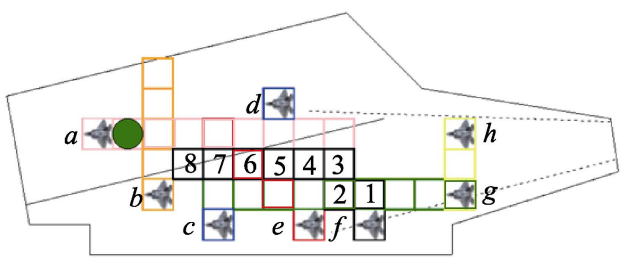

c. $t=8$

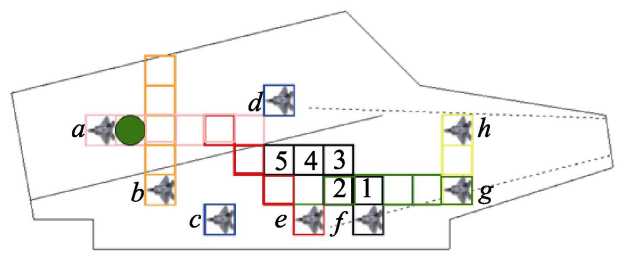

b. $t=5$

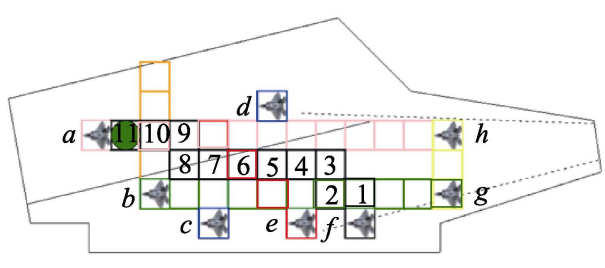

d. $t=11$

图 6 场景 3 中 PDQN 的仿真图

随机抽取收玫后的 80 组准确率, 计算平均准 确率和标准差. 如表 2 所示, SARSA 的准确率最高 (0.93), DQN 的准确率最低(0.78), Q-learning 和 PDQN 的准确率分别为 0.84 和 0.81 . 如表 3 所示, 随机抽取收玫后的 100 组无碰撞的路径长度, 计算 平均路径长度. 设置 3 个场景最短距离均为 11 个 网格. 其中 PDQN 所找到的平均路径最短, 且最接 近最短距离 11, DQN, Q-learning, SARSA 所对应的 平均路径长度分别为 $12.029,12.985,12.981$.

\subsection{2 场景 1}

场景 1 中, 4 种方法分别训练 25000 回合耗时 $3.0 \sim 3.5 \mathrm{~h}$ 且均在 $0.5 \mathrm{~h}$ 之内收玫. 图 7 所示为 Qlearning, SARSA, DQN 和 PDQN 的准确率. 其中, SARSA 的效果最好, DQN 的效果最差, 且 4 种方
法的收玫速度相差不大, 即自主学习能力相差不 大. 图 8 所示为各方法的平均奖励值. 由图 8 可看

表 2 不同方法在各个场景准确率

\begin{tabular}{lccc}
\hline \multicolumn{1}{c}{ 方法 } & 场景 1 & 场景 2 & 场景 3 \\
\hline PDQN & 0.81 & 0.76 & 0.75 \\
DQN $^{[11]}$ & 0.78 & 0.54 & 0.51 \\
Q-learning $^{[4]}$ & 0.84 & 0.58 & 0.55 \\
SARSA $^{[5]}$ & 0.93 & 0.58 & 0.59 \\
\hline
\end{tabular}

表 3 不同方法在各个场景路径长度

\begin{tabular}{llll}
\hline \multicolumn{1}{c}{ 方法 } & 场景 1 & 场景 2 & 场景 3 \\
\hline PDQN & 11.771 & 11.614 & 11.356 \\
DQN $^{[11]}$ & 12.029 & 12.125 & 12.585 \\
Q-learning $^{[4]}$ & 12.985 & 18.124 & 18.374 \\
SARSA $^{[5]}$ & 12.981 & 18.245 & 18.651 \\
\hline
\end{tabular}



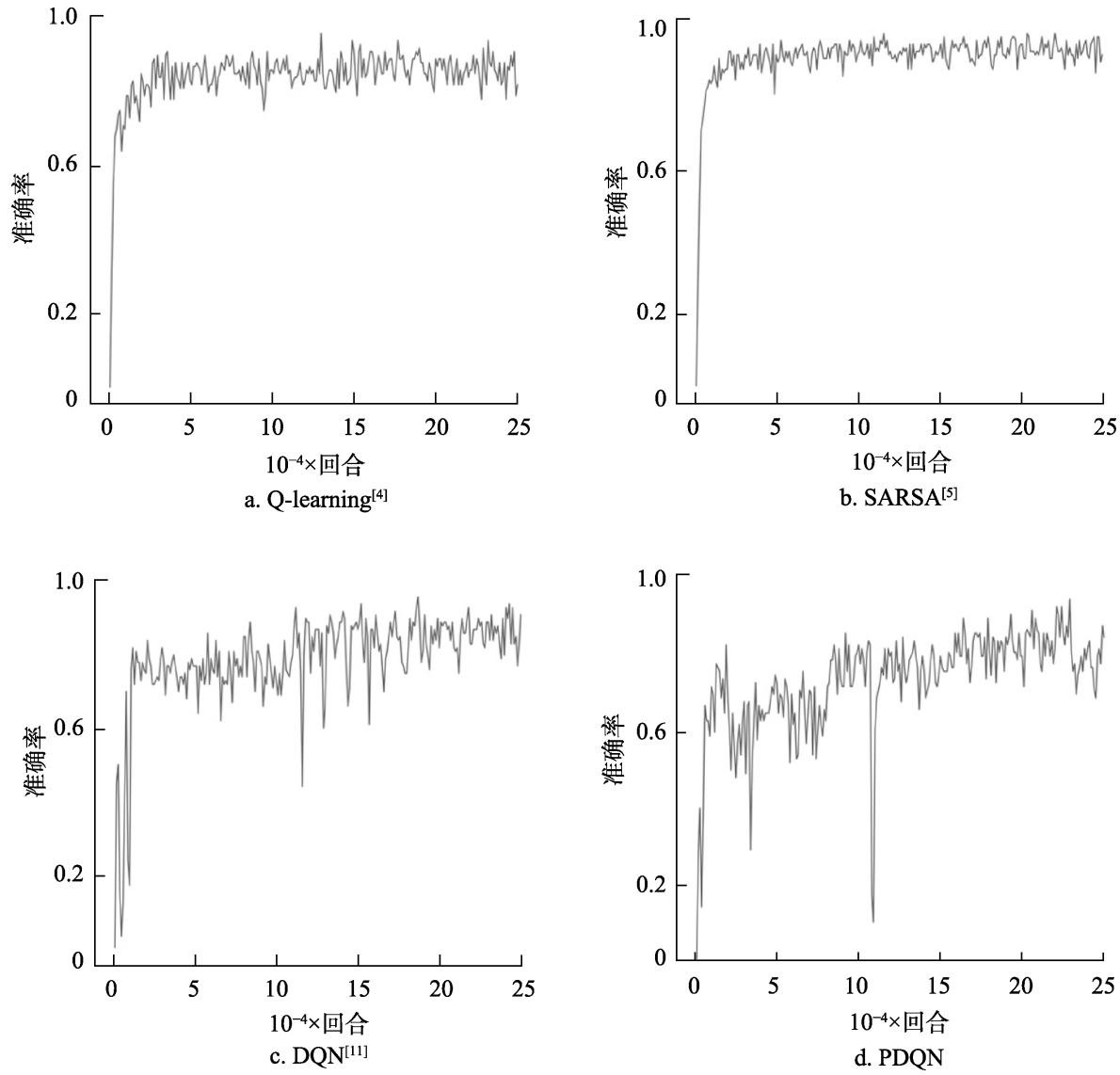

图 7 场景 1 准确率
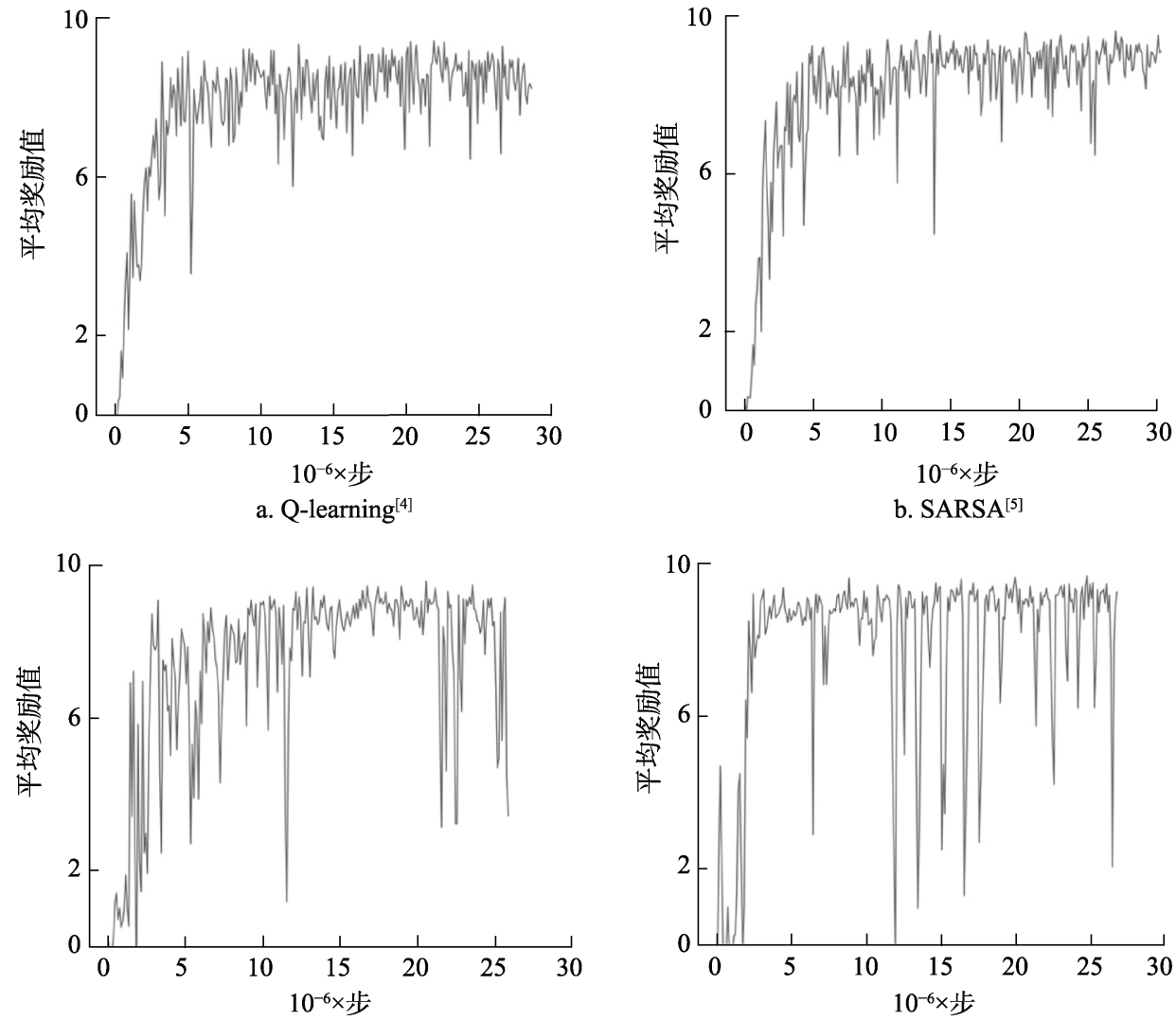

c. $\mathrm{DQN}^{[11]}$

图 8 场景 1 平均奖励值 
出, 各方法的平均奖励值为 8 9, 即舰载机所选择 的路线的合理性相差不大.

\subsection{3 场景 2}

图 9 所示为场景 2 中 Q-learning, SARSA, DQN 和 PDQN 的准确率. 如图 $9 \mathrm{a}$ 和图 $9 \mathrm{~b}$ 所示, 在高动 态障碍物环境中 Q-learning 和 SARSA 非常难收玫, 即分别训练 60000 回合耗时 $7 \sim 8 \mathrm{~h}$, 且均在 $3.5 \mathrm{~h}$ 后收敛. 而 DQN 和 PDQN 分别训练 25000 回合耗 时 3.0 3.5 h, 且均在 $0.5 \mathrm{~h}$ 之内收敛. 如表 2 所示, PDQN 的准确率最高 $(0.76)$, 而 DQN 的准确率最低 (0.54). Q-learning 和 SARSA 的准确率都为 0.58 . 如 表 3 所示, PDQN 所找到的平均路径最短，和场景 1 相比 PDQN 和 DQN 并没有因为复杂环境平均路径 长度发生较大的变化, Q-learning 和 SARSA 因为复 杂环境路径长度发生了较大的变化。DQN, Q-learning, SARSA 所对应的平均路径长度分别为 $12.125,18.124,18.245$. 图 10 所示为场景 2 中各方 法的平均奖励值. 其中, PDQN 的平均奖励值在 9 左右, DQN 则在 6 左右, Q-learning 和 SARSA 的平 均奖励值均在 4 以下, 即利用 PDQN 舰载机所选择 的路线更加合理. 综上所述, PDQN 相比于其他 3
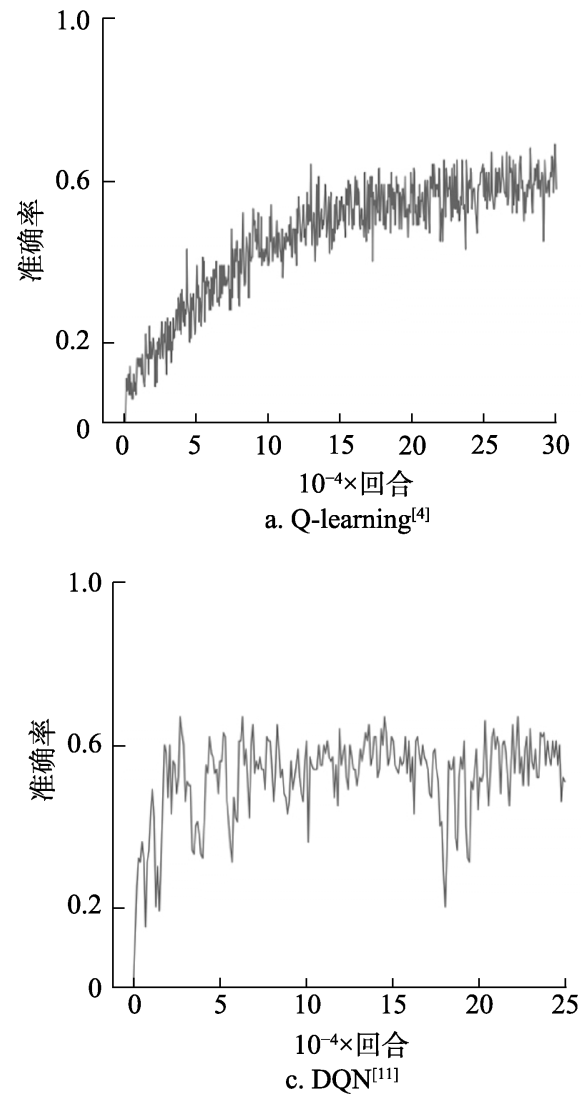

种方法更加有效、训练收玫后更稳定、所选路线更 加合理、自主学习能力更强. 在场景 2 中, PDQN 的标准差最小，即收玫后更加稳定.

\subsection{4 场景 3}

Q-learning, SARSA, DQN 和 PDQN 在场景 3 中的准确率如图 11所示. 其中, PDQN 的效果最好. 在场景 3 中 Q-learning 和 SARSA 同样训练 60000 回合耗时 $7 \sim 8 \mathrm{~h}$, 且均在 $3.5 \mathrm{~h}$ 之后收玫. 而 DQN 和 PDQN 分别训练 25000 回合耗时 3.0 3.5 h, 且 均在 $0.5 \mathrm{~h}$ 内收玫. 如表 2 所示, PDQN 的准确率最 高(0.75), 而 DQN 的准确率最低(0.51). Q-learning 和 SARSA 的准确率分别为 0.55 和 0.59. 如表 3 所 示, 场景 3 中 PDQN 所找到的平均路径最短, 而 DQN, Q-learning 以及 SARSA 的平均路径分别为 $12.585,18.374,18.651$. 图 12 所示为场景 3 中各个 方法的平均奖励值. 其中, PDQN 的平均奖励值为 8 , DQN 为 6, Q-learning 和 SARSA 均为 4. 即 PDQN 所选择的舰载机路线更加合理.

各方法在不同场景下的标准差如图 13 所示, 场景 1 中 SARSA 的标准差最小, 收玫后方法更加 稳定; 场景 2 和场景 3 中 PDQN 的标准差最小, 即
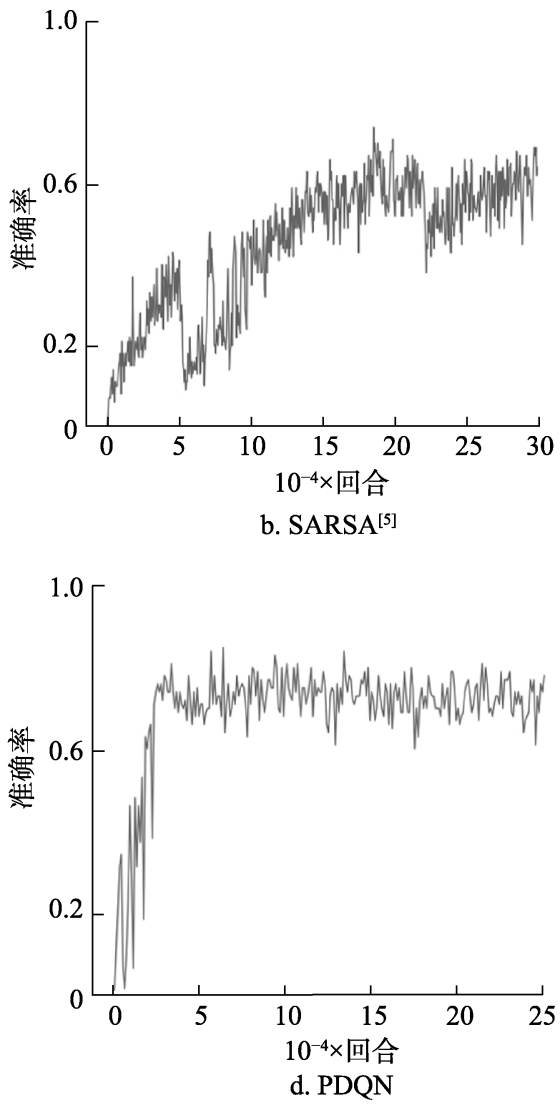

图 9 场景 2 准确率 

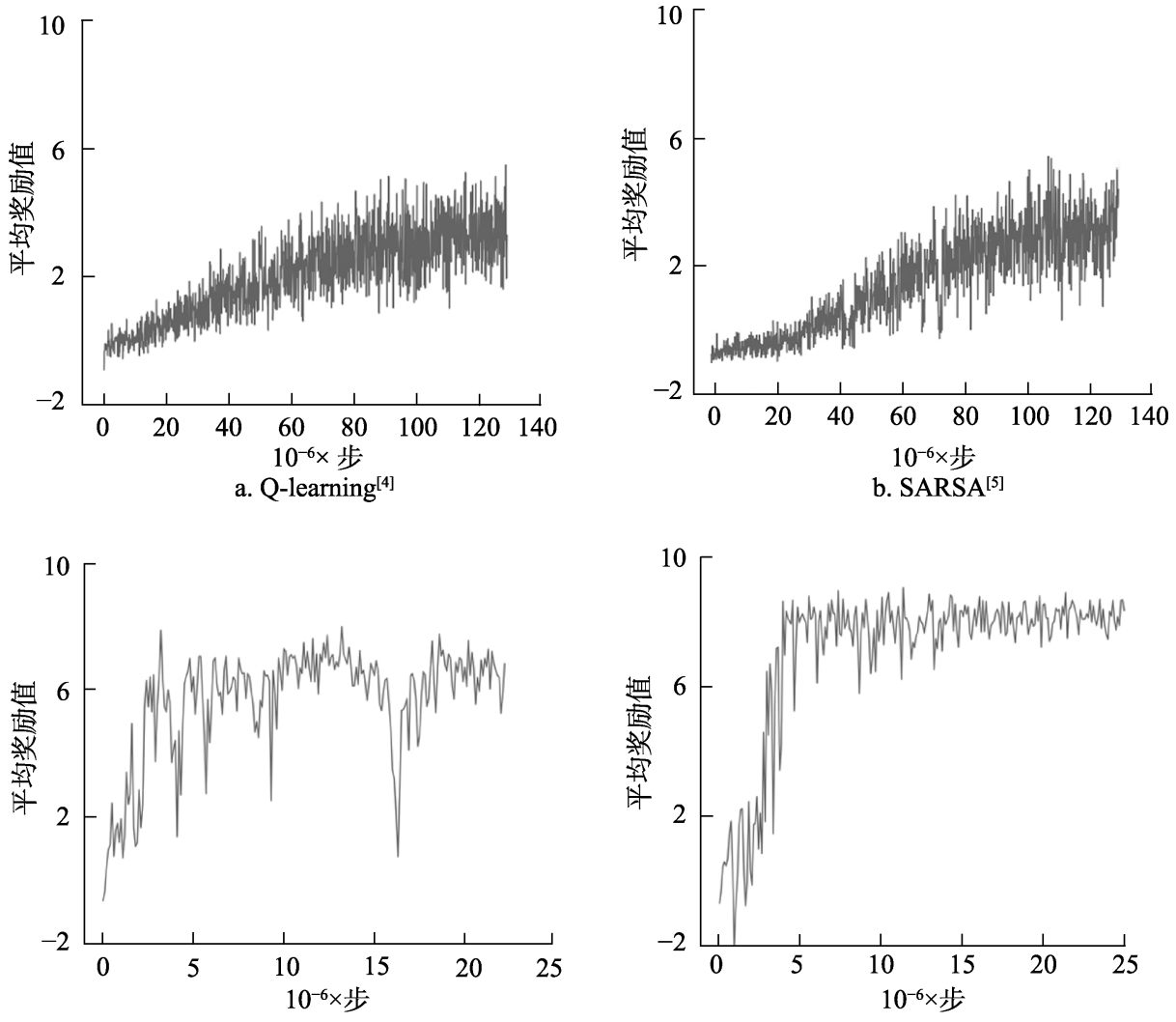

c. $\mathrm{DQN}^{[11]}$

d. PDQN

图 10 场景 2 平均奖励值
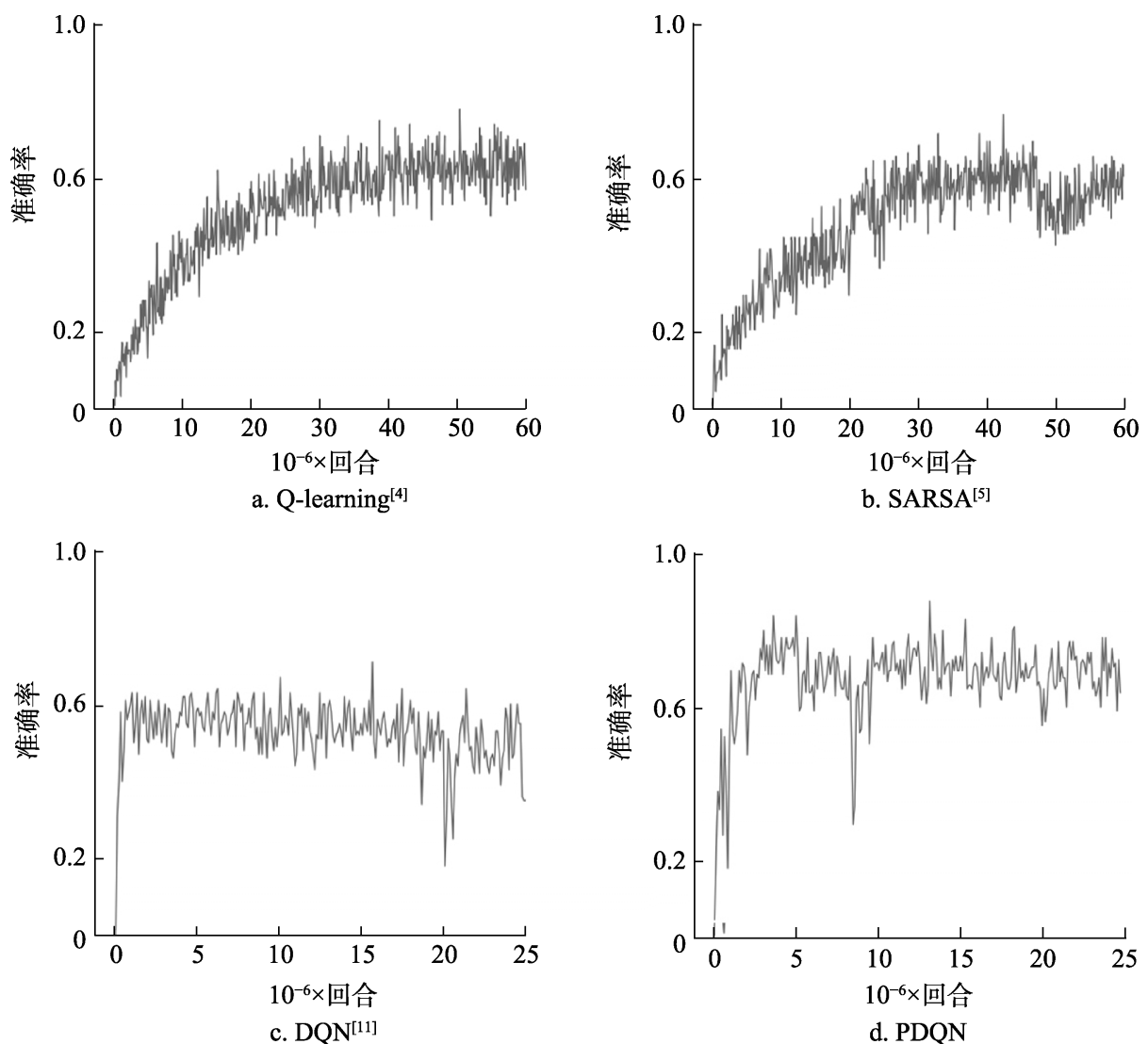

图 11 场景 3 准确率 

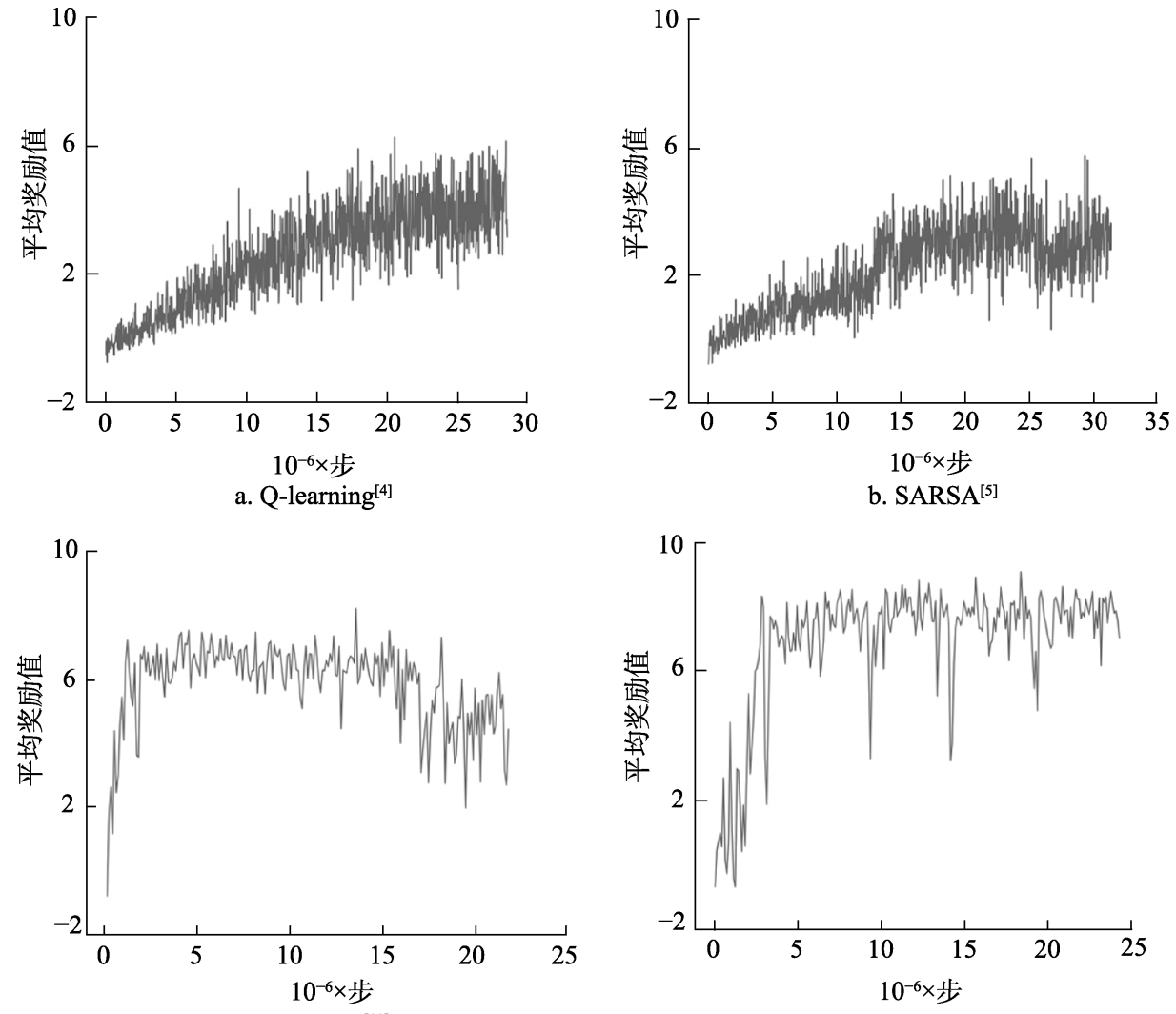

图 12 场景 3 平均奖励值

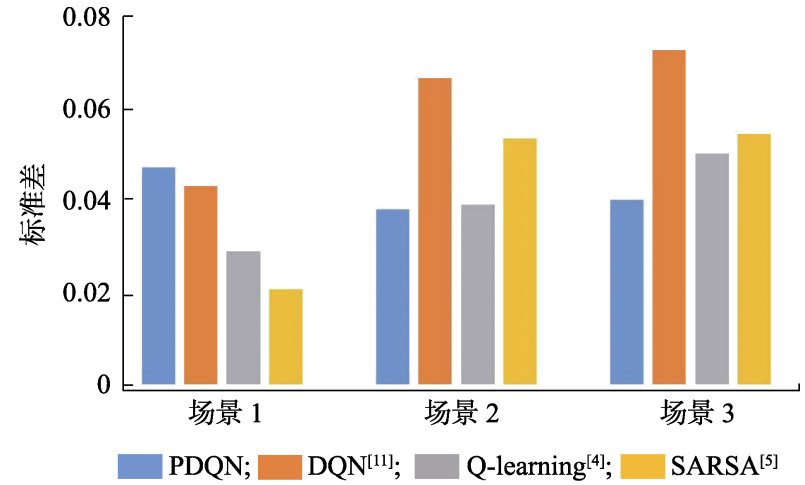

图 13 各方法在不同场景下的标准差

收玫后更加稳定. 综上所述, PDQN 比其他方法更 加有效、训练收敛后更稳定性、所选路线更加合理、 自主学习能力更强.

\section{4 结 语}

针对航母甲板动态避障这一研究问题，本文 首先深人分析了动态场景对于路径避障的技术挑 战. 在此基础上，提出了一种基于深度强化学习的 舰载机动态避障方法. 该方法将原研究问题进一 步细化为航母甲板作业场景建模、动态障碍物的轨 迹预测、基于奖惩机制的智能体动作反馈等 3 个子
问题. 针对 3 个子问题，利用多智能体建模、最小 二乘法和深度强化学习构建了航母甲板作业模型 和作业要素的路径规避方法, 解决了传统避障方 法需要大量数据训练且泛化能力差等问题, 提高 路径规划对动态环境的适应能力以及甲板作业方 案的灵活性. 通过 3 个场景的仿真实验, 验证了本 文方法在准确率、收玫速度、路径长度、平均奖励 值、稳定性上都有很好的表现.

\section{参考文献(References):}

[1] Bellman R. Dynamic programming and Lagrange multipliers [J]. Proceedings of the National Academy of Sciences of the United Stated America, 1956, 42(10): 767-769

[2] Werbos P J. Advanced forecasting methods for global crisis warning and models of intelligence[J]. General Systems, 1977, XXII: 25-38

[3] Sutton R S, Barto A G. Reinforcement learning: an introduction [M]. Cambridge: MIT Press, 1998

[4] Watkins C J C H, Dayan P. Technical note: Q-learning[J]. Machine Learning, 1992, 8(3/4): 279-292

[5] Rummery G A, Niranjan M. On-line Q-learning using connectionist systems[R] //Technical Report, vol. 37. England: Technical Report, 1994

[6] Bertsekas D P, Tsitsiklis J N. Neuro-dynamic programming: an 
overview[C] //Proceedings of the 34th IEEE Conference on Decision and Control. Los Alamitos: IEEE Computer Society Press, 1995: 560-564

[7] Thrun S. Monte Carlo POMDPs[C] //Proceedings of the 12th International Conference on Neural Information Processing Systems. Cambridge: MIT Press, 1999: 1064-1070

[8] Kocsis L, Szepesvári C. Bandit based Monte-Carlo planning[C] //Proceedings of the European Conference on Machine Learning. Heidelberg: Springer, 2006: 282-293

[9] Lewis F L, Vrabie D. Reinforcement learning and adaptive dynamic programming for feedback control[J]. IEEE Circuits and Systems Magazine, 2009, 9(3): 32-50

[10] Silver D, Lever G, Heess N, et al. Deterministic policy gradient algorithms[C] //Proceedings of the 31st International Conference on Machine Learning. Lille: PMLR Press, 2014: 387-395

[11] Mnih V, Kavukcuoglu K, Silver D, et al. Human-level control through deep reinforcement learning[J]. Nature, 2015, 518(7540): 529-533

[12] Silver D, Huang A, Maddison C J, et al. Mastering the game of go with deep neural networks and tree search[J]. Nature, 2016, 529(7587): 484-489

[13] Mnih V, Badia A P, Mirza M, et al. Asynchronous methods for deep reinforcement learning[C] //Proceedings of the International Conference on Machine Learning. Lille: PMLR Press, 2016: 1928-1937

[14] Lozano-Pérez T, Wesley M A. An algorithm for planning collision-free paths among polyhedral obstacles[J]. Communications of the ACM, 1979, 22(10): 560-570

[15] Barraquand J, Latombe J C. Robot motion planning: a distributed representation approach[J]. The International Journal of Robotics Research, 1991, 10(6): 628-649

[16] Hoffmann G, Waslander S, Tomlin C. Quadrotor helicopter trajectory tracking control[C] //Proceedings of the AIAA Guidance, Navigation, and Control Conference and Exhibit. Reston: AIAA.org, 2008: 7410

[17] Bohlin R, Kavraki L E. Path planning using lazy PRM[C] //Proceedings of the IEEE International Conference on Robot- ics and Automation. Los Alamitos: IEEE Computer Society Press, 2000: 521-528

[18] Hwang Y K, Ahuja N. A potential field approach to path planning[J]. IEEE Transactions on Robotics and Automation, 1992, 8(1): 23-32

[19] Bruce J, Veloso M M. Real-time randomized path planning for robot navigation[C] //Proceedings of the Robot Soccer World Cup. Heidelberg: Springer, 2002: 288-295

[20] Stentz A. Optimal and efficient path planning for partially known environments[M]. Heidelberg: Springer, 1997: 203-220

[21] Jaradat M A K, Al-Rousan M, Quadan L. Reinforcement based mobile robot navigation in dynamic environment[J]. Robotics and Computer-Integrated Manufacturing, 2011, 27 (1): 135-149

[22] Yan C, Xiang X J. A path planning algorithm for UAV based on improved Q-learning[C] //Proceedings of the 2nd International Conference on Robotics and Automation Sciences. Los Alamitos: IEEE Computer Society Press, 2018: 1-5

[23] Bouhamed O, Ghazzai H, Besbes H, et al. Q-learning based routing scheduling for a multi-task autonomous agent[C] //Proceedings of the 62nd IEEE International Midwest Symposium on Circuits and Systems. Los Alamitos: IEEE Computer Society Press, 2019: 634-637

[24] Chen Y F, Everett M, Liu M, et al. Socially aware motion planning with deep reinforcement learning[C] //Proceedings of the IEEE/RSJ International Conference on Intelligent Robots and Systems. Los Alamitos: IEEE Computer Society Press, 2017: 1343-1350

[25] Everett M, Chen Y F, How J P. Motion planning among dynamic, decision-making agents with deep reinforcement learning[C] //Proceedings of the IEEE/RSJ International Conference on Intelligent Robots and Systems. Los Alamitos: IEEE Computer Society Press, 2018: 3052-3059

[26] Tang K Q, Fu H Q, Jiang H, et al. Reinforcement learning for robots path planning with rule-based shallow-trial[C] //Proceedings of the 16th IEEE International Conference on Networking, Sensing and Control. Los Alamitos: IEEE Computer Society Press, 2019: 340-345 